\section{(6) OPEN ACCESS}

\title{
Ethnic differences in the association of QRS duration with ejection fraction and outcome in heart failure
}

\author{
Crystel M Gijsberts, ${ }^{1,2}$ Lina Benson, ${ }^{3}$ Ulf Dahlström, ${ }^{4,5}$ David Sim, ${ }^{6}$ Daniel P S Yeo, ${ }^{7}$ \\ Hean Yee Ong, ${ }^{8}$ Fazlur Jaufeerally, ${ }^{9},{ }^{10}$ Gerard K T Leong, ${ }^{11}$ Lieng H Ling, ${ }^{12,13}$ \\ A Mark Richards, ${ }^{12,13,14,15}$ Dominique P V de Kleijn, ${ }^{1,2,12,14}$ Lars H Lund, $^{16,17}$ \\ Carolyn S P Lam $6,10,12,14$
}

For numbered affiliations see end of article.

\section{Correspondence to} Dr Carolyn S P Lam, National Heart Centre Singapore, Singhealth, 5 Hospital Drive, Singapore 169609, Singapore; carolyn_lam@nuhs.edu.sg

LHL and CSPL contributed equally to this study.

Received 23 December 2015 Revised 12 May 2016 Accepted 11 June 2016 Published Online First 11 July 2016

\section{SLinked}

- http://dx.doi.org/10.1136/ heartjnl-2016-309760

\section{CrossMark}

To cite: Giisberts $C M$

Benson L, Dahlström U, et al. Heart

2016:102:1464-1471.

\section{ABSTRACT}

Background QRS duration (QRSd) criteria for device therapy in heart failure (HF) were derived from predominantly white populations and ethnic differences are poorly understood.

Methods We compared the association of QRSd with ejection fraction (EF) and outcomes between 839 Singaporean Asian and 11221 Swedish white patients with HF having preserved EF (HFPEF)and HF having reduced EF (HFREF) were followed in prospective population-based HF studies.

Results Compared with whites, Asian patients with HF were younger (62 vs 74 years, $p<0.001$ ), had smaller body size (height 163 vs $171 \mathrm{~cm}$, weight 70 vs $80 \mathrm{~kg}$, both $\mathrm{p}<0.001$ ) and had more severely impaired $\mathrm{EF}$ (EF was $<30 \%$ in $47 \%$ of Asians vs $28 \%$ of whites). Overall, unadjusted QRSd was shorter in Asians than whites (101 vs $104 \mathrm{~ms}, \mathrm{p}<0.001$ ). Lower EF was associated with longer QRSd $(p<0.001)$, with a steeper association among Asians than whites

$\left(p_{\text {interaction }}<0.001\right)$, independent of age, sex and clinical covariates (including body size). Excluding patients with left bundle branch block (LBBB) and adjusting for clinical covariates, QRSd was similar in Asians and whites with HFPEF, but longer in Asians compared with whites with $\operatorname{HFREF}(p=0.001)$. Longer QRSd was associated with increased risk of HF hospitalisation or death (absolute 2-year event rate for $\leq 120$ ms was $40 \%$ and for $>120$ ms it was 52\%; HR for 10 ms increase of QRSd was 1.04 (1.03 to 1.06), $p<0.001)$, with no interaction by ethnicity.

Conclusion We found ethnic differences in the association between EF and QRSd among patients with HF. QRS prolongation was similarly associated with increased risk, but the implications for ethnicity-specific QRSd cut-offs in clinical decision-making require further study.

\section{INTRODUCTION}

QRS prolongation (QRS duration (QRSd) $\geq 120 \mathrm{~ms}$ ) is an ECG characteristic of left ventricular (LV) remodelling occurring in $14 \%-47 \%$ of patients with heart failure $(\mathrm{HF}){ }^{1}$ It is related to reduction in LV ejection fraction (EF) and poor prognosis in $\mathrm{HF}^{2}$ QRSd cut-offs are used to guide device therapy decisions in $\mathrm{HF}$ with reduced $\mathrm{EF}$ (HFREF). ${ }^{3}$ However, the vast majority of prior data on QRSd in HF has been derived from studies in white patient populations. ${ }^{4}$ Ethnic differences in
ECG characteristics, and specifically in QRSd, have been described among adults without $\mathrm{HF}^{5}$ However in patients with HF, it is unclear if ethnic differences in QRSd exist or whether the relationships of QRSd with EF and prognosis are modified by ethnicity.

We sought to compare QRSd and its association with EF with prognosis between Asian and white patients with HF from population-based HF cohorts in Singapore ${ }^{6}$ and Sweden, ${ }^{7}$ respectively. Given that general-population Asian adults have a narrower QRS complex compared with generalpopulation white adults (possibly related to smaller heart sizes in Asians), ${ }^{8}$ we hypothesised that among patients with HF, Asians would also have narrower QRS complexes for a given EF. We further hypothesised that there may be important differences in the prognostic impact of QRS prolongation between Asian and white patients with HF.

\section{METHODS}

\section{Study population}

Our study population consisted of patients with HF from two contemporary population-based observational HF studies recruiting patients with $\mathrm{HF}$ regardless of EF, from either in-hospital (hospitalisation with primary diagnosis of HF) or outpatient (visit related to HF management) settings: Asian patients in the Singapore Heart Failure Outcomes and Phenotypes (SHOP) cohort ${ }^{6}$ and white patients in the Swedish Heart Failure Registry ${ }^{9}$ (SwedeHF).

The SHOP study enrolled patients with HF from all public institutions across the nation of Singapore and included a total of 1084 patients enrolled between June 2010 and July 2014. The SHOP protocol has been previously described in detail. ${ }^{6}$ Similarly, the SwedeHF study enrolled patients with HF across the nation of Sweden, beginning in $2000 .^{10}$ For the current analysis, we applied the same enrolment time frame to the SwedeHF cohort as in the SHOP cohort and thus only included 20073 patients from SwedeHF who were enrolled from 2010 (excluding the first 30987 patients enrolled from 2000 to 2009).

We excluded patients with missing EF (70 Asians and 3215 whites), missing QRSd ( $\mathrm{n}=6$ Asians and $\mathrm{n}=4918$ whites), missing or inconsistent information on left bundle branch block (LBBB) $(n=49$ Asians and $n=519$ whites), device therapy $(n=73$ Asians and $n=0$ whites (already excluded due to 
missing QRS, which was not registered in case of device therapy)) or who died during hospitalisation or with missing follow-up information ( $n=62$ Asians and $n=200$ whites), thereby leaving 839 Asian and 11221 white patients in the final study population.

\section{Ethics statement}

This study conforms to the Declaration of Helsinki. All SHOP participants provided written informed consent. For the SwedeHF registry, individual patient consent was not required, but patients were informed of entry into the national registry and allowed to opt out. Both cohorts were approved by the responsible ethics committees as described earlier for both cohorts. ${ }^{69}$

This study is performed and reported in accordance with the STROBE guidelines for observational research. ${ }^{11}$

\section{Ethnicity}

For SwedeHF, ethnicity on individual basis was not available; all Swedish patients were considered to be of white ethnic background. In the SHOP cohort, ethnicity was known at the individual level (from Singapore national ID registration): 63\% was of Chinese ethnic background, 27\% Malaysian, 9\% Indian and $1 \%$ of other ethnic background. All SHOP participants were considered to be of Asian ethnicity for the purpose of this study.

\section{HFREF and HFPEF}

In both cohorts, HFREF was defined as clinical features of HF with an ECG EF $<50 \%$, as used in most previous studies. ${ }^{12}$ The EF of patients with HFREF was further categorised into 40\%$49 \%$, 30\%-39\% and $<30 \%$. HF with preserved EF (HFPEF) was defined as clinical features of HF and an ECG EF $\geq 50 \%$.

\section{Patient characteristics}

Baseline patient characteristics were gathered from SwedeHF and the Swedish Patient Registry for the Swedish cohort. For SHOP, baseline characteristics were collected through a case record form specifically designed for the cohort (as described earlier). ${ }^{6}$

In SHOP, QRSd was available from automatic ECG readings and confirmed by independent manual measurement by blinded trained staff. In SwedeHF, the QRSd was determined by the reporting clinician and not further specified whether measured manually or digitally.

For both cohorts, a history of coronary artery disease (CAD) was defined as either a history of stable CAD (as assessed by a physician), myocardial infarction, percutaneous coronary intervention (PCI) or coronary artery bypass surgery.

\section{Outcomes}

Outcomes were collected through standardised protocols in both cohorts. Mortality was obtained by checking the national death registry of Singapore and the Population Registry in Sweden. HF hospitalisations were obtained from hospital records in Singapore and ICD-10 code registrations in the main (first) position of the Patient Registry in Sweden. Follow-up events were considered after dismissal from the index event.

\section{Statistical analysis}

Baseline characteristics were stratified by ethnicity. Continuous, normally distributed data were presented as means \pm SD and compared using a t-test. Non-normally distributed data were presented as medians with IQRs and compared using Kruskal-Wallis testing. Categorical data were reported as percentages and compared using $\chi^{2}$ testing.
Because of covariates that were potentially missing not-completely-at-random, covariates were imputed for multivariable analysis by means of single imputation with 10 iterations using all covariates in the two cohorts separately, using the 'MICE' package for R. ${ }^{13}$ Primary variables of interest and outcome measures were not imputed (EF, QRSd and events during follow-up).

We compared QRSd between the Asians and whites by EF strata (HFREF or HFPEF) using linear regression. Due to the non-normal distribution of residuals of QRSd in the linear regression model, it was analysed as $1 / \mathrm{QRS}$. The transformation method was determined by means of the Box-Cox transformation. ${ }^{14}$ We examined whether ethnicity modified the association between $\mathrm{EF}$ and $1 / \mathrm{QRSd}$ using interaction terms in a multivariable model.

Next, we determined how ethnicity affected 1/QRS separately in patients with HFPEF and patients with HFREF with and without LBBB in models adjusted for age, sex, EF (only for HFREF), height, body mass index (BMI), diastolic blood pressure, hypertension, diabetes, smoking, history of CAD, PCI, coronary artery bypass grafting, valve surgery, atrial fibrillation or flutter (AF), stroke, peripheral arterial disease or chronic obstructive pulmonary disease (COPD), New York Heart Association (NYHA) class, $\mathrm{N}$-terminal pro-brain natriuretic peptide (NTproBNP), creatinine clearance (calculated with CockcroftGault formula), haemoglobin, duration of HF ( $>6$ vs $\leq 6$ months), heart rate, medications ( $\beta$ blocker, ACE inhibitor, angiotensin II receptor blocker, diuretic, statin, antiplatelet therapy) and LBBB (only for analyses including patients with LBBB). These covariates were pre-specified based on clinical relevance.

Subsequently, we assessed the effect of cumulatively adding each covariate to the model containing the primary variable (ethnicity). Ethnicity-specific back-transformed fitted values of $1 / \mathrm{QRSd}$ were calculated for all subgroups of patients from the multivariable models.

Finally, we examined the ethnicity-specific association of QRSd (which had a linear relation with outcome and thus required no transformation) with a composite end point of HF hospitalisation and all-cause mortality in Cox regression models for HFPEF and HFREF separately. The association of QRSd with outcome was tested in a univariable model (including interaction analysis) and in a model including all covariates as mentioned above. The additional analysis for outcome HF was censored at death. The HRs of QRSd (reference value 120) for Asians and whites were visualised using the coxsimLinear and simGG functions from the 'simPH' 15 package for R.

All analyses were performed using Rstudio (J Allaire. RStudio: integrated development environment for R. Boston, Massachusetts, USA, 2012.) and R software (R Core Team. R: A Language and Environment for Statistical Computing, 2013.) for statistical computing (V.3.1.2). A p value of $<0.05$ was considered to be statistically significant and all $\mathrm{p}$ values were two-sided.

\section{RESULTS}

\section{Baseline characteristics by ethnicity}

Compared with white patients with HF, Asian patients with HF were younger (62 vs 74 years, $\mathrm{p}<0.001$ ), had smaller body size (height 163 vs $171 \mathrm{~cm}$, weight 70 vs $80 \mathrm{~kg}$ and BMI 26.3 vs 27.1, all $\mathrm{p}<0.001$ ) and had more severely impaired EF (EF was $<30 \%$ in $47 \%$ of Asians vs $28 \%$ of whites) but less severe NYHA classification and lower NTproBNP levels (median 2044 vs $2700 \mathrm{pg} / \mathrm{mL}, \mathrm{p}<0.001$ ) (table 1). A history of CAD was more common among Asians $(53 \%$ vs $45 \%, \mathrm{p}<0.001)$, but $\mathrm{AF}$ and valve surgery were more common among whites $(50 \%$ vs $23 \%$ and $5 \%$ vs $1 \%$ respectively; both $\mathrm{p}<0.001$ ) (table 1 ). 
Table 1 Baseline characteristics of patients with HF stratified by ethnicity

\begin{tabular}{|c|c|c|c|c|c|}
\hline & Asian & Percentage missing & White & Percentage missing & p Value \\
\hline$n$ & 839 & & 11221 & & \\
\hline Age (years, mean $\pm S D$ ) & $61.8 \pm 12.2$ & 0 & $73.5 \pm 12.3$ & 0 & $<0.001$ \\
\hline Sex (female \%) & 24.2 & 0 & 38.4 & 0 & $<0.001$ \\
\hline Height $(\mathrm{cm}$, mean $\pm S D)$ & $162.5 \pm 8.6$ & 1.5 & $171.3 \pm 9.9$ & 48.5 & $<0.001$ \\
\hline Weight $(\mathrm{kg}$, mean $\pm S D)$ & $69.6 \pm 15.8$ & 1.4 & $79.5 \pm 18.7$ & 7.1 & $<0.001$ \\
\hline BMI $\left(\mathrm{kg} / \mathrm{m}^{2}\right.$, mean $\left.\pm \mathrm{SD}\right)$ & $26.3 \pm 5.4$ & 1.9 & $27.1 \pm 5.6$ & 49.1 & $<0.001$ \\
\hline $\mathrm{SBP}(\mathrm{mm} \mathrm{Hg}$, mean $\pm \mathrm{SD})$ & $125.9 \pm 22.5$ & 0.2 & $128.2 \pm 21.2$ & 1.1 & $<0.001$ \\
\hline $\mathrm{DBP}(\mathrm{mm} \mathrm{Hg}$, mean $\pm \mathrm{SD})$ & $71.9 \pm 13.0$ & 0.2 & $73.6 \pm 12.4$ & 1.2 & 0.003 \\
\hline Hypertension (\%) & 72.6 & 1.2 & 54.9 & 1.7 & $<0.001$ \\
\hline Diabetes (\%) & 55.7 & 0.2 & 23.7 & 0.2 & $<0.001$ \\
\hline Smoking (\%) & & 0.2 & & 18.7 & $<0.001$ \\
\hline Non-smoker & 46.6 & & 43.0 & & \\
\hline Ex-smoker & 29.7 & & 42.4 & & \\
\hline Current smoker & 23.7 & & 14.6 & & \\
\hline Creatinine clearance $^{*}(\mathrm{~mL} / \mathrm{min}$, mean $\pm \mathrm{SD})$ & $66.3 \pm 38.8$ & 4.4 & $68.3 \pm 33.7$ & 7.8 & 0.117 \\
\hline \multicolumn{6}{|l|}{ Medical history } \\
\hline CAD (\%) & 53.4 & 4.3 & 45.0 & 0 & $<0.001$ \\
\hline $\mathrm{PCl}(\%)$ & 19.9 & 1.5 & 16.6 & 0 & 0.018 \\
\hline CABG (\%) & 13.7 & 0.6 & 22.4 & 0 & $<0.001$ \\
\hline Valve surgery (\%) & 0.7 & 0.6 & 5.2 & 0.6 & $<0.001$ \\
\hline Atrial fibrillation/flutter (\%) & 23.3 & 0.8 & 50.0 & 0 & $<0.001$ \\
\hline Stroke $(\%)$ & 10.3 & 0.2 & 17.2 & 0 & $<0.001$ \\
\hline PAD (\%) & 4.2 & 0.8 & 10.4 & 0 & $<0.001$ \\
\hline COPD (\%) & 8.3 & 0.5 & 18.6 & 0 & $<0.001$ \\
\hline \multicolumn{6}{|l|}{ Heart failure characteristics } \\
\hline Duration of $\mathrm{HF}>6$ months (\%) & 40.2 & 0.7 & 39.3 & 1.1 & 0.613 \\
\hline $\mathrm{EF}(\%)$ & & 0 & & 0 & $<0.001$ \\
\hline$=>50$ & 22.2 & & 24.3 & & \\
\hline $40-49$ & 11.2 & & 21.3 & & \\
\hline $30-39$ & 19.7 & & 26.6 & & \\
\hline$<30$ & 47.0 & & 27.8 & & \\
\hline NYHA (\%) & & 1.3 & & 35.1 & $<0.001$ \\
\hline 1 & 26.9 & & 11.8 & & \\
\hline II & 58.1 & & 49.1 & & \\
\hline III & 14.0 & & 35.1 & & \\
\hline IV & 1.0 & & 3.9 & & \\
\hline Heart rate $(\mathrm{bpm}$, mean $\pm S D)$ & $75.4 \pm 14.3$ & 0 & $74.5 \pm 15.9$ & 2.1 & 0.124 \\
\hline LBBB (\%) & 4.6 & 0 & 16.0 & 0 & $<0.001$ \\
\hline NTproBNP (pg/mL, median (IQR)) & $2044(808,4266)$ & 0 & $2700(1178,5858)$ & 57.7 & $<0.001$ \\
\hline Haemoglobin $(\mathrm{g} / \mathrm{L}, \mathrm{mean} \pm \mathrm{SD})$ & $13.0 \pm 2.1$ & 0 & $13.2 \pm 1.8$ & 0 & $<0.001$ \\
\hline \multicolumn{6}{|l|}{ Medication use } \\
\hline$\beta$-blocker (\%) & 93.6 & 0 & 87.4 & 0.4 & $<0.001$ \\
\hline ACE inhibitor (\%) & 66.0 & 0 & 65.2 & 0.4 & 0.656 \\
\hline ARB (\%) & 19.4 & 0 & 21.9 & 1.0 & 0.110 \\
\hline Diuretics (\%) & 94.9 & 0 & 78.1 & 0.6 & $<0.001$ \\
\hline Statins (\%) & 88.8 & 0 & 48.2 & 0.3 & $<0.001$ \\
\hline Antiplatelet (\%) & 82.5 & 0 & 49.8 & 0.4 & $<0.001$ \\
\hline
\end{tabular}

\section{QRSd in Asians and whites with HFPEF and HFREF}

The distribution of QRSd between Asians and whites is depicted in figure 1. The overall relationship of QRSd with EF differed by ethnicity: in Asians, a reduction in EF was related to a steeper increase of QRSd compared with whites ( $p_{\text {interaction }}$ $<0.001$ in the multivariable model). Similar results were obtained when LBBB was excluded.
As described in our methods section, QRSd was analysed as 1/ QRSd due to non-normally distributed residuals in the regression analysis. In the total population, QRSd was shorter in Asians than whites in univariable analysis (back-transformed values from 1/QRS: 101 vs $104 \mathrm{~ms}, \mathrm{p}<0.001$ ), both in HFPEF (91 vs $96 \mathrm{~ms}, \mathrm{p}<0.001)$ and HFREF (104 vs $106 \mathrm{~ms}, \mathrm{p}=0.012$ ). LBBB was more common in white compared with Asian patients (16 vs 

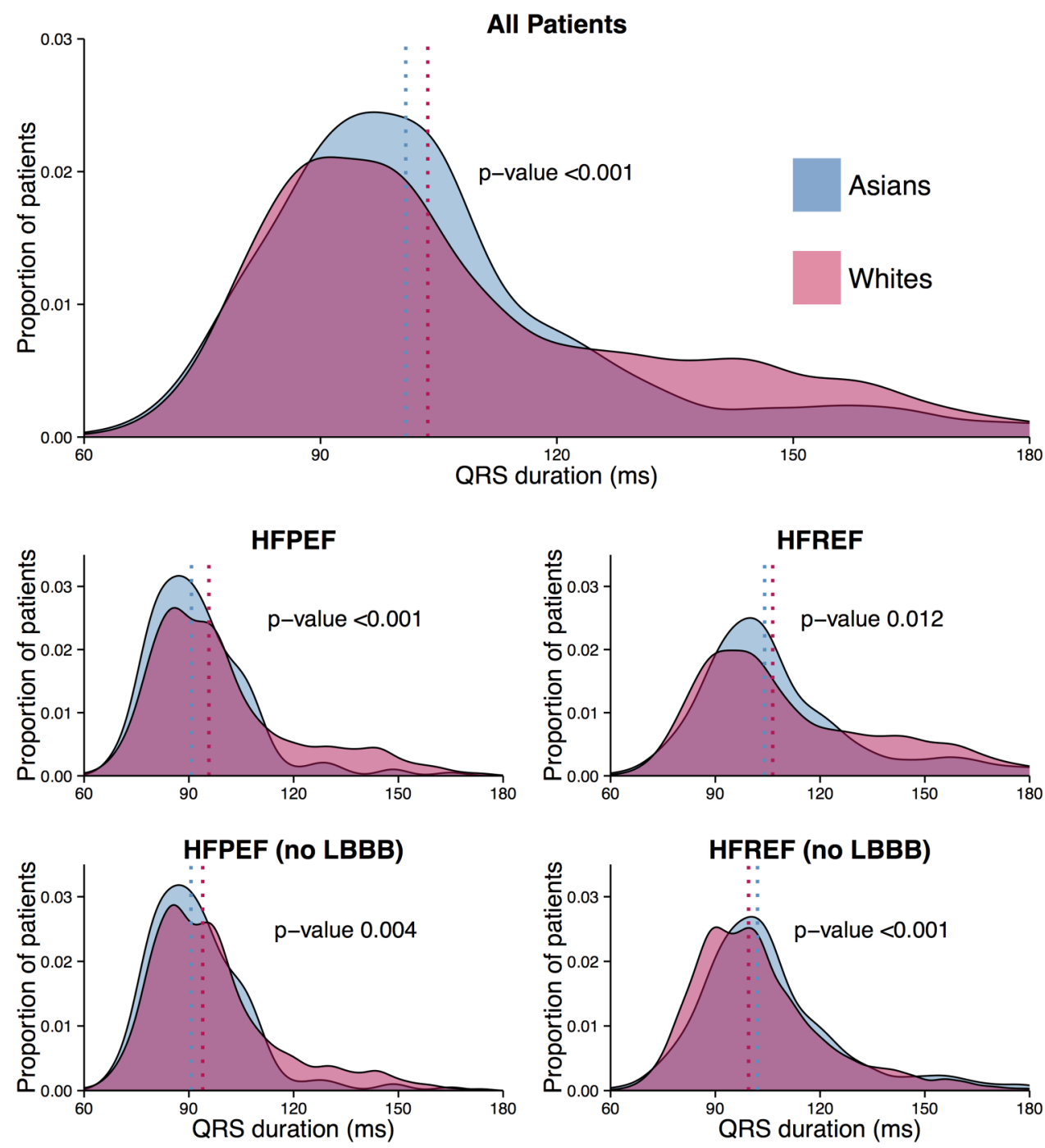

Figure 1 Asians are depicted in blue and whites are depicted in pink. The top distribution plot shows QRS duration (QRSd) distribution for all patients, regardless of ejection fraction (EF) or left bundle branch block (LBBB). The middle plots give the QRSd distribution for Asians and whites, stratified by EF group (HFPEF or HFREF). The bottom plots show QRSd distribution for patients without LBBB. $p$ Values are given for the comparison between Asians and whites. HFPEF, heart failure with preserved EF; HFREF, heart failure with reduced EF.

$5 \%, \mathrm{p}<0.001)$. After excluding patients with LBBB, QRSd was found to be shorter in Asian compared with white patients with HFPEF (91 vs $94 \mathrm{~ms}, \mathrm{p}=0.006$ ) but longer in Asian compared with white patients with HFREF (102 vs $100 \mathrm{~ms}, \mathrm{p}<0.001)$.

After multivariable adjustment, there was no longer a significant difference in QRSd among Asians and whites with HFPEF, whereas QRSd was now significantly longer in Asian compared with white patients with HFREF (105 vs $103 \mathrm{~ms}, \mathrm{p}=0.027$ ). Fitted QRSd values derived from the multivariable models are summarised in table 2.

The effect of the addition of each covariate on the relationship between ethnicity and QRSd is shown in figure 2 . Covariates that strongly influenced the relationship between ethnicity and QRSd included LBBB, EF, sex, age and height.

\section{Prognostic value of QRSd for HF hospitalisation and mortality in HFPEF and HFREF}

During a median follow-up duration of 445 days, 2941 HF hospitalisations (298 Asians, 2643 whites) and 2358 deaths (127 Asians, 2231 whites) occurred.

The association of baseline QRSd (modelled as a continuous variable) with the composite end point of HF hospitalisation or all-cause death was assessed in both Asian and white patients with HFPEF and HFREF in four separate analyses (visualised in figure 3). Among all patients, the multivariable adjusted HR for a $10 \mathrm{~ms}$ increase in QRSd was 1.04 (95\% CI 1.03 to 1.06 , $\mathrm{p}<0.001)$. The HR for a $10 \mathrm{~ms}$ increase in QRSd was 1.07 (95\% CI 0.88 to $1.31, \mathrm{p}=0.533$ ) in Asians with HFPEF, $1.02(95 \%$ CI 0.99 to $1.06, p=0.180)$ in whites with HFPEF, 1.01 (95\% CI 0.94 to $1.05, \mathrm{p}=0.861$ ) in Asians with HFREF and 1.06 (95\% CI 1.04 to $1.07, \mathrm{p}<0.001)$ in whites with HFREF. No significant interactions were found for this composite end point. A separate multivariable analysis for HF hospitalisations showed similar results; no interactions were found between ethnicity and QRSd for this event among all patients $\left(p_{\text {interaction }}=0.183\right)$ and separately for patients with HFPEF $\left(p_{\text {interaction }}=0.347\right)$ and patients with HFREF ( $\left.p_{\text {interaction }}=0.158\right)$. These results indicated that there are no significant differences in the prognostic value of QRSd prolongation between the ethnicities.

Interactions and three-way interactions between the most prominent baseline differences (AF, COPD and diabetes) and QRSd (and ethnicity in case of three-way interactions) in relation with outcome were analysed and found to be non- 
Table 2 Values of QRSd by ethnicity before and after multivariable adjustment

\begin{tabular}{|c|c|c|c|c|c|}
\hline \multirow[b]{2}{*}{ Patients } & \multicolumn{2}{|l|}{ Unadjusted } & \multicolumn{2}{|l|}{ Adjusted } & \multirow[b]{2}{*}{$\begin{array}{l}\text { p Value } \\
\text { (adjusted) }\end{array}$} \\
\hline & $\begin{array}{l}\text { QRSd }(95 \% \mathrm{CI}) \\
\text { Asians }\end{array}$ & $\begin{array}{l}\text { QRSd }(95 \% \mathrm{CI}) \\
\text { Whites }\end{array}$ & $\begin{array}{l}\text { QRSd }(95 \% \mathrm{Cl}) \\
\text { Asians }\end{array}$ & $\begin{array}{l}\text { QRSd }(95 \% \mathrm{CI}) \\
\text { Whites }\end{array}$ & \\
\hline All & 100.8 (99.5 to 102.2 ) & $103.6(103.2$ to 104.0$)$ & $104.9(103.6$ to 106.4$)$ & $103.3(103.0$ to 103.6$)$ & 0.027 \\
\hline HFPEF & 90.7 (89.0 to 92.6 ) & 95.7 (95.1 to 96.4 ) & 95.3 (92.8 to 98.0 ) & 95.4 (94.8 to 96.0$)$ & 0.945 \\
\hline HFREF & 104.1 (102.6 to 105.7$)$ & $106.4(105.9$ to 106.9$)$ & $108.5(106.9$ to 110.2$)$ & $106.1(105.7$ to 106.5$)$ & 0.006 \\
\hline HFPEF (no LBBB) & 90.6 (88.9 to 92.4 ) & $94.0(93.4$ to 94.6$)$ & 93.8 (91.3 to 96.4$)$ & 93.7 (93.2 to 94.3 ) & 0.967 \\
\hline HFREF (no LBBB) & $102.1(100.7$ to 103.6$)$ & 99.5 (99.1 to 99.9 ) & $101.7(100.1$ to 103.4$)$ & 99.6 (99.2 to 99.9 ) & 0.014 \\
\hline
\end{tabular}

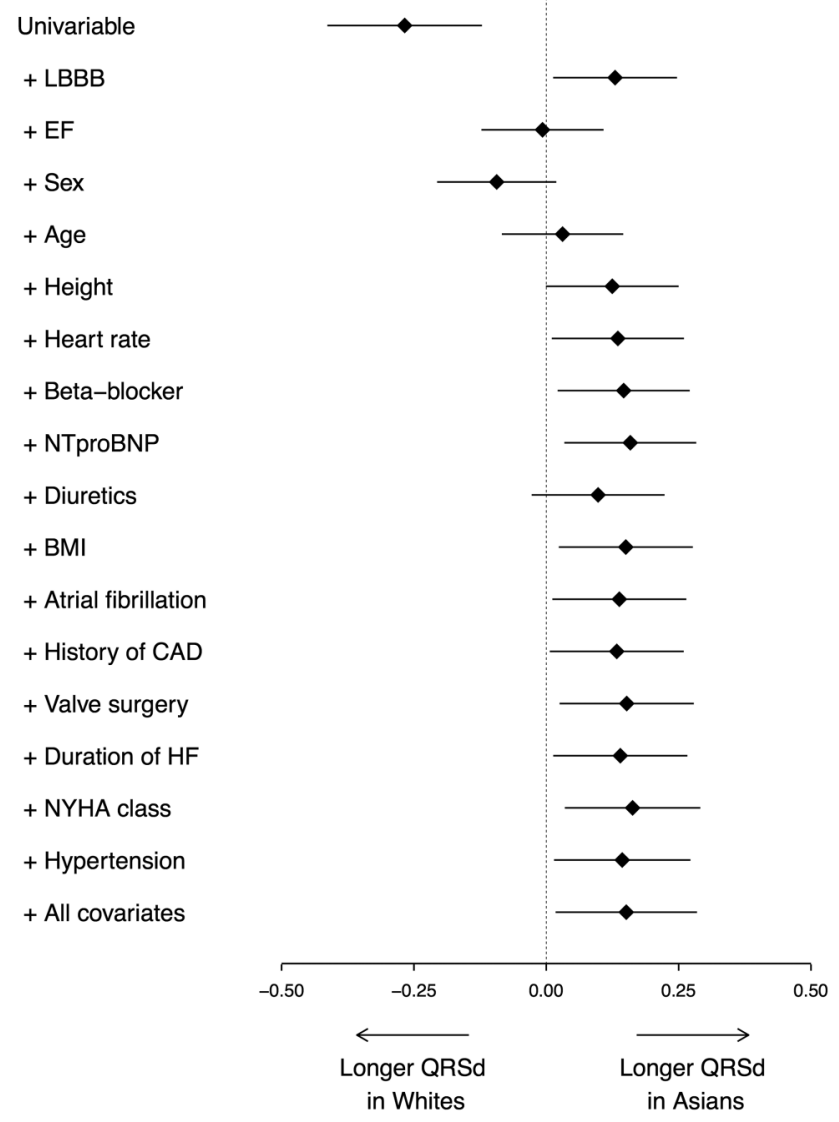

Figure 2 The points and lines display the point estimate $(\beta)$ and $95 \%$ $\mathrm{Cls}$ of the association of Asian versus white ethnicity with 1/QRS duration (QRSd). The $\beta$ value represents the difference between Asian and white QRSd. The top estimate displays the univariable association of ethnicity with 1/QRSd. Subsequently, all covariates that were used in multivariable analysis were cumulatively added to the model (in the order of significance in the final model; most significant covariates first). Variables with a significant relation with $1 / Q R S d$ are shown separately in the figure; for each of those models the association of ethnicity with 1/QRSd is shown. The bottom estimate is the result of the full multivariable model; variables with a non-significant relation with $1 /$ QRSd are also included in this model, but not shown separately. BMI, body mass index; $C A D$, coronary artery disease; $E F$, ejection fraction; $H F$, heart failure; LBBB, left bundle branch block; NTproBNP, N-terminal pro-brain natriuretic peptide; NYHA, New York Heart Association. significant. This indicates that these comorbidities did not change the ethnicity-specific relation of QRSd with outcome.

\section{DISCUSSION}

These are the first data comparing QRSd and its correlates with prognostic significance between Asian and white patients with HF from parallel prospective population-based cohorts. Ethnicity significantly modified the relationship between EF and QRSd in HF, with a steeper association between QRS prolongation and EF reduction among Asians than whites. While QRSd was generally shorter in Asians than whites in unadjusted comparisons, after multivariable adjustment, there was no longer any ethnic difference in QRSd among patients with HFPEF, whereas among patients with HFREF, QRSd was longer in Asians compared with whites. There were no ethnic differences in the association between QRS prolongation and adverse outcomes.

QRS prolongation in patients with $\mathrm{HF}$ has been examined among whites, blacks and Hispanics, ${ }^{16}$ showing shorter QRSd in blacks than whites with HFREF. ${ }^{17}$ No prior studies have evaluated QRSd in Asians versus whites with HF or the association between ethnicity, QRSd and outcomes in HFPEF versus HFREF. Extrapolating from findings in the general population, we hypothesised that Asian patients with HF would have narrower QRSd compared with white patients with $\mathrm{HF}$ at any given EF. While we indeed found shorter QRSd in Asian patients in unadjusted comparisons, after carefully adjusting for baseline differences between cohorts, we were surprised to find more severe QRS prolongation in Asians compared with whites with HFREF, contrary to our hypothesis. To the extent that EF is a major determinant of $\mathrm{QRSd},{ }^{2}$ this is also consistent with the steeper increase in QRSd for a given decline in EF and thus potentially a greater sensitivity to adverse effects of remodelling among Asians compared with whites.

The mechanisms underlying the greater increases in QRSd with decreases in EF in Asians are unclear. One intriguing consideration relates to ethnic differences in what may be considered 'normal' QRSd in Asians versus whites. In general population adults without HF, a shorter QRSd has been described in Asians compared with whites. ${ }^{8} 1819$ This may relate to intrinsically smaller heart sizes in Asians. ${ }^{20}$ Height, as a surrogate of heart size, ${ }^{21}$ was directly correlated to QRSd $(\mathrm{p}<0.001)$ in the current study. In fact, after adjusting for 

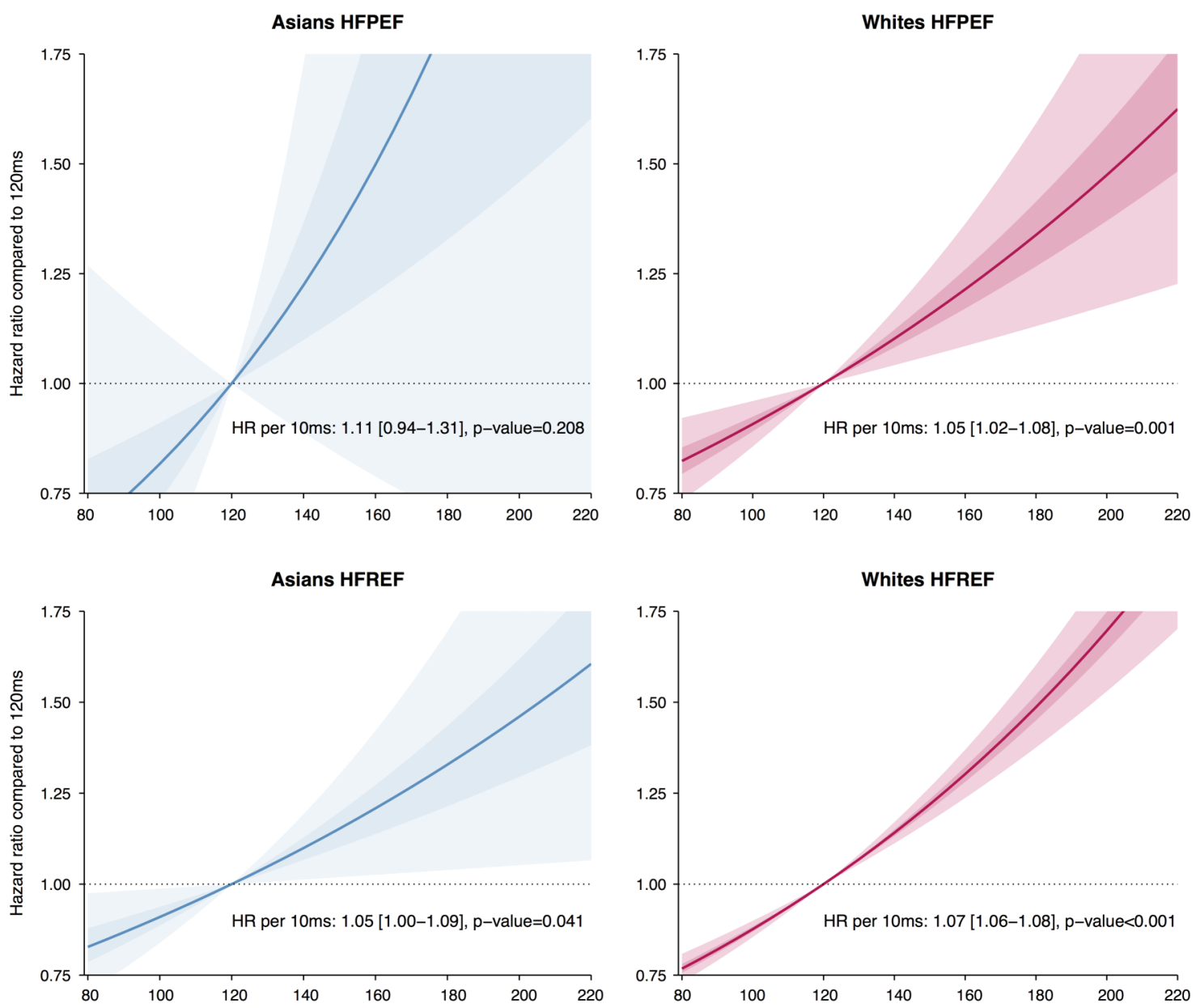

Figure 3 Cox regression survival estimates (hazard ratios) are displayed for Asian (in blue, left panel) and white (in red, right panel) patients with heart failure (HF), using $120 \mathrm{~ms}$ as the reference value. The top two plots show the relation of QRS duration (QRSd) with outcome for patients with $\mathrm{HF}$ with preserved ejection fraction (HFPEF), the bottom two for patients with HF with reduced ejection fraction (HFREF). The outcome is HF hospitalisation or all-cause death. The plotted estimates are derived from univariable analysis. The shaded areas represent the $50 \% \mathrm{Cl}$ (darkest area) and the $95 \% \mathrm{Cl}$ (lighter area). No significant interaction between QRSd and ethnicity was found for outcome (no significant differences between the slopes of the lines for Asians and whites).

height and other covariates (figure 2), the difference in QRSd between Asians and whites with HFPEF was rendered nonsignificant (table 2), suggesting that differences in body and heart size may indeed account for ethnic differences in QRSd when EF is preserved.

Another consideration relates to what is considered 'normal' EF in Asians versus whites. In the general population Multi-Ethnic Study of Atherosclerosis (MESA) cohort, cardiac MRI showed a significantly higher EF of $72 \%$ for Chinese than whites (EF 68\%). ${ }^{22}$ Similarly, the EchoNormal study ${ }^{20}$ showed that 'normal' $\mathrm{EF}$ is considerably higher in East Asians than in whites (lower reference value for $\mathrm{EF} 56 \%$ vs $50 \%$ in 50 -year-old men and $57 \%$ vs $51 \%$ in 50 -year-old women). A given low EF measurement in HFREF (eg, EF 30\%) may therefore represent a greater EF decline (greater LV eccentric remodelling) in Asians than whites, which may explain the greater extent of QRSd prolongation in Asians than in whites with HFREF. In other words, more severe QRS prolongation in Asians with HFREF may reflect greater scar burden from adverse LV remodelling with a greater reduction in EF from a higher 'normal' value in Asians compared with whites.

Interestingly, similar considerations have been applied to explain sex differences in response to device therapy in HFREF, where, using the same QRSd/EF cut-off, women derive greater benefit from device therapy than men. ${ }^{23}$ This sex difference has been related to known differences in 'normal' QRSd and EF between women and men. ${ }^{24}$

QRS prolongation is known to be a predictor of mortality in $\mathrm{HF}$, as shown in prior studies ${ }^{9}$ and confirmed in the current. One other study directly compared the prognostic value of QRSd among ethnicities although in a different population (healthy general population); the MESA study ${ }^{25}$ found no significant interaction between ethnicity (whites, blacks, Hispanics, Chinese) and QRSd in the general population without HF. We, therefore, extend this to the HF population in the current study, showing no modifying effect of ethnicity on the association of QRSd and outcomes.

While our findings are notable, we acknowledge that our observational data from two separate cohorts cannot provide conclusive evidence for different QRSd cut-offs for Asians and whites and cannot be extrapolated to response to treatment such as cardiac resynchronisation therapy. We had limited statistical power to assess outcomes in the HFPEF subgroups and were unable to assess the ethnicity-specific relationships of QRSd with outcome between the HF types (ie, test for threeway interactions among ethnicity, QRSd and HF type). 
The divergence of the direction of the association of ethnicity with QRSd between patients with HFPEF and patients with HFREF renders a systematic measurement error between SwedeHF and SHOP highly unlikely.

While SwedeHF and SHOP are large universal real-life registries in which EF was systematically collected for all patients, more detailed aspects from echocardiography such as LV dimensions or mass unfortunately were unavailable in SwedeHF. Therefore, we could not perform a direct comparison of the relationship between QRSd and LV size or mass in the combined cohort and it remains possible that differences in heart size mediated the observed differences in QRSd.

Data collection occurred through separate although similar protocols in SwedeHF and SHOP; thus, differences in data collection are unavoidable. However the majority of the merged data were standard variables in HF that are uniformly assessed globally and data collection periods of the cohorts were deliberately matched. While we cannot have perfect matching on all fields, ours is the only combined database of population-based HF from European and Asian countries. Also, the median follow-up of 445 days may not be long enough to reveal prognostic differences. Results may therefore be considered hypothesis-generating and deserve further study in contemporaneous parallel prospective studies using identical protocols in different countries.

We carefully adjusted our cross-sectional and survival analyses for all available baseline variables; however, residual confounding by unmeasured characteristics cannot be excluded. Separate analyses for patients with LBBB alone were not feasible due to low patient numbers in those groups.

\section{CONCLUSIONS}

These first data comparing QRSd between Asian and white patients with HF from parallel prospective population-based cohorts showed that ethnicity significantly modified the

\section{Key messages}

\section{What is already known on this subject?}

- QRS prolongation is an ECG measurement contributing to an indication for device therapy in patients with heart failure (HF). However, QRS duration (QRSd) cut-offs were derived from predominantly white populations and ethnic differences are poorly understood.

\section{What might this study add?}

- We compared the association of QRSd with ejection fraction (EF) and outcomes between 839 Singaporean Asian and 11221 Swedish white patients with HF having preserved and reduced EF were followed in prospective population-based HF studies.

- Overall, QRSd was longer among whites than among Asians. However, we observed that with the deterioration of the EF, QRSd increased more in Asians than in whites. The prognostic value of QRSd did not differ significantly between Asians and whites.

\section{How might this impact on clinical practice?}

- The ethnicity-specific relation between EF and QRSd is something that clinicians should be aware of and it deserves more attention in clinical research. In particular, it should be investigated whether ethnicity-specific QRSd cut-off values for device therapy decision-making are necessary. relationship between EF and QRSd in HF, with a steeper association between QRS prolongation and EF reduction among Asians compared with whites. Further studies are needed to determine whether ethnicity-specific QRSd cut-offs for clinical decisionmaking regarding device therapy in HF should be considered.

\section{Author affiliations}

${ }^{1}$ ICIN-Netherlands Heart Institute, Utrecht, The Netherlands

${ }^{2}$ Laboratory of Experimental Cardiology, University Medical Centre Utrecht, Utrecht, The Netherlands

${ }^{3}$ Department of Clinical Science and Education, Karolinska Institutet, Stockholm, Sweden

${ }^{4}$ Department of Cardiology, Linköping University, Linköping, Sweden

${ }^{5}$ Department of Medical and Health Sciences, Linköping University, Linköping, Sweden

${ }^{6}$ National Heart Centre, Singhealth, Singapore, Singapore

${ }^{7}$ Department of Cardiology, Tan Tock Seng Hospital, Singapore, Singapore

${ }^{8}$ Department of Cardiology, Khoo Teck Puat Hospital, Singapore, Singapore

${ }^{9}$ Department of Medicine/Cardiology, Singapore General Hospital, Singapore,

Singapore

${ }^{10}$ Duke-NUS, Singapore, Singapore

${ }^{11}$ Department of Cardiology, Changi General Hospital, Singapore, Singapore

${ }^{12}$ Yong Loo Lin School of Medicine, National University, Singapore, Singapore

${ }^{13}$ Cardiac Department, National University Health System, Singapore, Singapore

${ }^{14}$ Cardiovascular Research Institute, National University, Singapore, Singapore

${ }^{15}$ Christchurch Heart Institute, University of Otago, Christchurch, New Zealand

${ }^{16}$ Department of Medicine, Karolinska Institutet, Karolinska University Hospital,

Stockholm, Sweden

${ }^{17}$ Department of Cardiology, Karolinska Institutet, Karolinska University Hospital, Stockholm, Sweden

Contributors DS, DPSY, HYO, FJ, GKTL, LiHL, AMR, UD, LaHL and CSPL provided substantial contributions to data acquisition. LB and CMG performed data analysis and DPVdK, LaHL and CSPL assisted in interpreting the results. The manuscript was drafted by CMG, CSPL, LaHL and LB. DS, DPSY, HYO, FJ, GKTL, LiHL, AMR, UD and DPVdK critically revised the manuscript for important intellectual content. All authors provided approval to publish the manuscript in its current form. All authors have agreed to be accountable for all aspects of the work in ensuring that questions related to the accuracy or integrity of any part of the work are appropriately investigated and resolved. CSPL is guarantor of this article.

Funding This work was supported by grants to Lars Lund's Institution from Swedish Research Council (grant 2013-23897-104604-23); Swedish Heart Lung Foundation (grants 20100419, 20120321); Stockholm County Council (grants 20110120, 20140220); Swedish Society of Medicine (grant 174111) and Boston Scientific. This work was additionally supported by a Strategic grant from the Royal Netherlands Academy of Arts and Sciences to the Interuniversity Cardiology Institute of the Netherlands, ICIN to DPVdK; National University Singapore Startup grant to DPVdK, Singapore National Medical Research Council Centre Grant to AMR, DPVdK and CSPL and the ATTRaCT, SPF 2014/003 grant BMRC to CSPL, DPVdK and AMR. CSPL is supported by a Clinician Scientist Award from the National Medical Research Council of Singapore and has received research support from Boston Scientifc, Medtronic and Vifor Pharma.

\section{Competing interests None declared.}

Ethics approval Multisite IRB Singapore and multisite Ethics Committee in Sweden. Provenance and peer review Not commissioned; externally peer reviewed.

Open Access This is an Open Access article distributed in accordance with the Creative Commons Attribution Non Commercial (CC BY-NC 4.0) license, which permits others to distribute, remix, adapt, build upon this work non-commercially, and license their derivative works on different terms, provided the original work is properly cited and the use is non-commercial. See: http://creativecommons.org/ licenses/by-nc/4.0/

\section{REFERENCES}

1 Kashani A, Barold SS. Significance of QRS complex duration in patients with heart failure. J Am Coll Cardiol 2005;46:2183-92.

2 Sandhu R, Bahler RC. Prevalence of QRS prolongation in a community hospital cohort of patients with heart failure and its relation to left ventricular systolic dysfunction. Am J Cardiol 2004;93:244-6.

3 Brignole M, Auricchio A, Baron-Esquivias G, et al. 2013 ESC Guidelines on cardiac pacing and cardiac resynchronization therapy: the Task Force on cardiac pacing and resynchronization therapy of the European Society of Cardiology (ESC). Developed in collaboration with the European Heart Rhythm Association (EHRA). Eur Heart J 2013;34:2281-329. 
4 Colvin M, Sweitzer NK, Albert NM, et al. Heart failure in non-Caucasians, women, and older adults: a white paper on special populations from the Heart Failure Society of America Guideline Committee. J Card Fail 2015;21:674-93.

5 Ambrosy AP, Gheorghiade M, Chioncel 0, et al. Global perspectives in hospitalized heart failure: regional and ethnic variation in patient characteristics, management, and outcomes. Curr Heart Fail Rep 2014;11:416-27.

6 Santhanakrishnan R, Ng TP, Cameron VA, et al. The Singapore Heart Failure Outcomes and Phenotypes (SHOP) study and Prospective Evaluation of Outcome in Patients with Heart Failure with Preserved Left Ventricular Ejection Fraction (PEOPLE) study: rationale and design. J Card Fail 2013;19:156-62.

7 Jonsson $A$, Edner $M$, Alehagen $U$, et al. Heart failure registry: a valuable tool for improving the management of patients with heart failure. Eur J Hear Fail J Work Gr Hear Fail Eur Soc Cardiol 2010;12:25-31.

8 Tan E, Xu CF, Liang F, et al. Association of ethnicity, age and body size with electrocardiographic values in the community. J Am Coll Cardiol 2014;63:A1636.

9 Lund LH, Jurga J, Edner M, et al. Prevalence, correlates, and prognostic significance of QRS prolongation in heart failure with reduced and preserved ejection fraction. Eur Heart J 2013;34:529-39.

10 Eklind-Cervenka M, Benson L, Dahlström U, et al. Association of candesartan vs losartan with all-cause mortality in patients with heart failure. JAMA 2011;305:175-82.

11 von Elm E, Altman DG, Egger M, et al. The Strengthening the Reporting of Observational Studies in Epidemiology (STROBE) statement: guidelines for reporting observational studies. PLoS Med 2007;4:e296.

12 Davis BR, Kostis JB, Simpson LM, et al. Heart failure with preserved and reduced left ventricular ejection fraction in the antihypertensive and lipid-lowering treatment to prevent heart attack trial. Circulation 2008;118:2259-67.

13 Van Buuren S, Groothuis-Oudshoorn K. Multivariate imputation by chained equations. J Stat Softw 2011;45:1-67.

14 G. E. P. Box DRC. An analysis of transformations. J R Stat Soc Ser B $1964 ; 26: 211-52$.
15 Gandrud C. simPH: an R package for showing estimates for interactive and nonlinear effects from Cox Proportional Hazard Models. SSRN Electron J 2015;65:1-20.

16 Hebert K, Quevedo HC, Tamariz L, et al. Prevalence of conduction abnormalities in a systolic heart failure population by race, ethnicity, and gender. Ann Noninvasive Electrocardiol 2012;17:113-22.

17 Ilkhanoff L, Soliman EZ, Ning H, et al. Factors associated with development of prolonged QRS duration over 20 years in healthy young adults: The coronary artery risk development in young adults study. J Electrocardiol 2012;45:178-84.

18 Macfarlane PW, Katibi IA, Hamde ST, et al. Racial differences in the ECG-selected aspects. J Electrocardiol 2014;47:809-14.

19 Mansi IA, Nash IS. Ethnic differences in electrocardiographic intervals and axes. J Electrocardiol 2001;34:303-7.

20 Echocardiographic Normal Ranges Meta-Analysis of the Left Heart Collaboration. Ethnic-specific normative reference values for echocardiographic LA and LV size, LV mass, and systolic function: The EchoNoRMAL Study. JACC Cardiovasc Imaging 2015;8:656-65

21 Pfaffenberger $S$, Bartko $P$, Graf A, et al. Size matters! Impact of age, sex, height, and weight on the normal heart size. Circ Cardiovasc Imaging 2013;6:1073-9.

22 Bahrami H, Kronmal R, Bluemke DA, et al. Differences in the incidence of congestive heart failure by ethnicity: the multi-ethnic study of atherosclerosis. Arch Intern Med 2008;168:2138-45.

23 Zabarovskaja S, Gadler F, Braunschweig F, et al. Women have better long-term prognosis than men after cardiac resynchronization therapy. Europace 2012;14:1148-55.

24 Rijnbeek PR, van Herpen G, Bots ML, et al. Normal values of the electrocardiogram for ages 16-90 years. J Electrocardiol 2014;47:914-21.

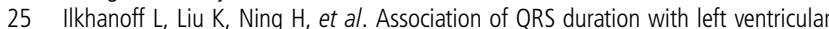
structure and function and risk of heart failure in middle-aged and older adults: The Multi-Ethnic Study of Atherosclerosis (MESA). Eur J Heart Fail 2012;14:1285-92. 